\title{
Caracterización del autoconcepto en una muestra de estudiantes universitarios de algunos programas de pregrado de la ciudad de
}

\section{(1)Psicogente ISSN 0124-0137 EISNN 2027-212X}

Artículo de Investigación Copyright 2018 by Psicogente

Correspondencia de autores: diana.montoya@ucaldas.edu.co victoria.pinilla@ucaldas.edu.co carmen.dussan@ucaldas.edu.co

Recibido: 25-11-16

Aceptado: 06-05-17

Publicado: 01-01-18
Manizales

\section{Self-concept characterization based on university students sampled of some undergraduate programs from Manizales}

\author{
Diana Marcela Montoya Londoño iD, Victoria Eugenia Pinilla Sepúlveda \\ Universidad de Caldas, Colombia; Universidad de Manizales, Ciudad de Manizales, Colombia \\ Carmen Dussán Luberth \\ Universidad de Caldas, Manizales, Colombia
}

Resumen

Objetivo: Caracterizar el autoconcepto en una muestra de estudiantes universitarios adscritos a dos universidades de la ciudad de Manizales y analizar el efecto de la disciplina y la formación profesional sobre este constructo.

Método:Se empleó el cuestionario AF5, se describieron las variables, se comparó el desempeño en las diferentes dimensiones del autoconcepto presentado por los estudiantes, discriminando entre semestres por programas y género. Para ello se trabajó la prueba $\mathrm{t} \mathrm{o}$ análisis de varianza (dependiendo del número de medias a comparar), o las pruebas no paramétricas correspondientes.

Resultados: Se encontró que para el caso de los hombres, a nivel de autoconcepto académico-laboral y social, los estudiantes del programa de Filosofía presentan menores promedios que los de las demás carreras evaluadas. Se evidenciaron menores promedios entre los estudiantes de Historia y Licenciatura en Ciencias Sociales, respecto a programas del área de la salud. En el caso de las mujeres, se hallaron diferencias significativas a nivel del autoconcepto académico-laboral entre las estudiantes de primer semestre del programa de Filosofía con un menor promedio, respecto a las mujeres del programa de Desarrollo Familiar

Conclusiones: Los estudiantes quedarán ubicados por encima del promedio de los baremos existentes para la prueba, lo que hace evidente la necesidad de realizar estudios de estandarización de la prueba para población universitaria colombiana.

Palabras Claves:

Autoconcepto, Relaciones interpersonales, Relaciones familiares. Fuente: DeCS, BIREME.

Abstract

Objective: This research sampled 375 university students belonging to two universities in Manizales during 2013-I in order to analyze self-concept, and the effects of discipline and professional training.

Method: The AF5 self-concept questionnaire was used. For this purpose, variables were described and characterized in the performance of self-concept related to academic-labor, social, emotional, family and physical dimensions and compared and differentiated based on semesters, program and genre. A T test was applied for variance analysis (depending on the number of averages to compare) or the corresponding non-parametric tests.

Results: It was found that men from the Philosophy program compared with the others from the evaluated programs, presented lower averages related to self-conception concerning academic, labor and social levels. Also when comparing a Bachelor's degree in Social Sciences and History, History students showed evident lower averages. On the other hand, female students in the first semester Philosophy program showed evident differences in self-conception concerning academic-labor compared to female students in the Family Development program.

Conclusion: Students will be considered above average in the existing scales for the testing, which makes evident the need to carry out standardization studies of the test for Colombian university population.

Key words:

Self-concept, Interpersonal relationships, Family relationships. Source: DeCS, BIREME. 


\section{INTRODUCCIÓN}

El autoconcepto es entendido como el conjunto de conceptos internamente consistentes y jerárquicamente organizados acerca de diferentes dimensiones humanas entre las cuales se reconocen los factores físico, social, emocional, académico y personal; se comprende como el concepto que una persona tiene acerca de sí misma. Puede definirse como la totalidad de los pensamientos y sentimientos que un individuo tiene acerca de sí mismo y es considerado como una actitud básica que condiciona el comportamiento del sujeto, el rendimiento escolar y la construcción de la personalidad (SauraCalixto, 1995; García \& Musitu, 2009; García, Sarmiento, \& Martínez, 2011; Ramírez, 2012; Salvador-Ferrer, 2012; Véliz-Burgos \& Apodaca-Urquijo, 2012a). En esta perspectiva, el autoconcepto se construye a través de las experiencias vitales y de las relaciones con el entorno, siendo muy relevantes tanto las respuestas de uno mismo, como de las de personas significativas (Vélez, Doner \& Sandoval, 2016).

El autoconcepto es el producto de la reflexión que el individuo tiene acerca sí mismo como un ser físico, social y espiritual (Musitu, Román \& Gracia, 1988; García \& Musitu, 2009; Malo, Bataller, Casas, Gras, \& González, 2011; Ibarra, Armenta \& Jacobo, 2014). Así entendido, representa una variable psicológica determinante en la vida del joven universitario, en su plan de vida y en la construcción de su identidad personal como ser humano y como profesional, que puede construirse y fortalecerse a partir de las interacciones que el estudiante establece en la familia, el entorno social, e incluso en el paso por la Universidad con otras personas que le resultan significativas (Cataño, Restrepo, Portilla \& Ramírez, 2008; Torres-Monje, 2016; García-Cruz, 2016). De hecho, algunos investigadores consideran que el autoconcepto se construye, y está conectado directamente, con variables educativas como la implicación del estudiante en el proceso de aprendizaje (Ramos-Díaz, Rodríguez-Fernández, Fernández-Zabala, Revuelta, \& Zuazagoitia, 2016).

La presente investigación tuvo como objetivo caracterizar el autoconcepto en una muestra de estudiantes universitarios adscritos a dos universidades de la ciudad de Manizales y analizar el efecto de la disciplina y la formación profesional sobre este constructo. Este problema de investigación se origina desde el interés en establecer las características de las diferentes dimensiones del autoconcepto en estudiantes de diferentes disciplinas, por considerar, que el dominio específico puede tener efecto en la forma como se configura y consolida en la persona el concepto que tiene acerca de sí misma.

En este sentido, diferentes investigaciones relacionan autoconcepto y desempeño profesional, de hecho, en algunos estudios para el caso de la enfermería, se señala una imagen pública de la profesión diversa e incongruente, en medio de la cual se evidencian problemas asociados a la forma como desde dicha profesión se deriva el autoconcepto y la identidad profesional, a partir de la imagen pública, que es en parte auto-creada por las(os) enfermeras(os) debido a su invisibilidad y 
su falta de discurso público (Hoeve, Jansen \& Roodbol, 2014), mientras que en profesiones como la educación, estudios realizados en China evidencian la consolidación del autoconcepto de educadores de museos en Ciencias a partir de la forma como desde esta profesión se construye un autoconcepto multifacético y contextual, a partir de la valoración que hacen las personas de las motivaciones en el trabajo y la responsabilidad y competencia laboral (Ji, 2015).

\section{MÉTODO}

\subsection{Diseño}

La investigación es de tipo transversal de carácter inferencial.

\subsection{Participantes}

Estudiantes de la Universidad de Caldas y de la Universidad de Manizales, matriculados en el período 2013-I en los primeros tres semestres o los últimos tres semestres en los programas de Historia, Licenciatura en Ciencias Sociales (los cuales se trabajaron como uno solo), Medicina, Psicología, Desarrollo familiar, Licenciatura en Filosofía y Letras y Licenciatura en Educación Básica con énfasis en Educación Física, Recreación y Deportes (la que será enunciada a partir de este punto como Licenciatura en Educación Física). Mediante un muestreo por conglomerados (Ospina, 2001) de tamaños diferentes (los cursos con estudiantes cuyo número de créditos aprobados a 2013-I correspondía a estar iniciando o finalizando la carrera) para estimación de medias, se obtuvo una muestra constituida por 375 personas, discriminadas como lo indica la Tabla 1. Se aclara que en algunos programas únicamente se trabajó con estudiantes mujeres u hombres, ya que el porcentaje de unos u otros en tales programas no permitía la comparación por géneros.

Tabla 1.

Muestra discriminada por género y semestre del estudiante

\begin{tabular}{|c|c|c|c|c|c|}
\hline \multirow[t]{2}{*}{ Programa } & \multicolumn{2}{|c|}{ Primeros semestres } & \multicolumn{2}{|c|}{ Últimos semestres } & \multirow[t]{2}{*}{ Total fila } \\
\hline & Femenino & Masculino & Femenino & Masculino & \\
\hline Historia y Lic. Ciencias Sociales & 24 & 31 & 14 & 16 & 85 \\
\hline Desarrollo familiar & 38 & 0 & 25 & 0 & 63 \\
\hline Lic. Ed. Física & 0 & 28 & 0 & 21 & 49 \\
\hline Lic. Filosofía y letras & 14 & 22 & 7 & 10 & 53 \\
\hline Medicina & 20 & 34 & 13 & 16 & 83 \\
\hline Psicología & 22 & 0 & 20 & 0 & 42 \\
\hline Total Columna & 118 & 115 & 79 & 63 & 375 \\
\hline
\end{tabular}

\subsection{Instrumentos}


En la presente investigación se utilizó el cuestionario AF5 (Autoconcepto Forma 5) (García \& Musitu, 2009\}).

El AF5, Autoconcepto Forma 5, es una escala para evaluar el autoconcepto construida por García \& Musitu (1999) de aplicación individual y colectiva. A la hora de diseñar el AF5, se definieron 6 ítems de cada una de las 5 dimensiones, resultando un cuestionario total de 30 ítems con estructura pentadimensional. El cuestionario se confeccionó sobre la base teórica del modelo multidimensional y jerárquico de la estructura del autoconcepto propuesta por los relevantes trabajos de Shavelson, Hubner y Stanton (1976). El proceso de administración dura 15 minutos aproximadamente, incluyendo la aplicación y la corrección. Tiene como finalidad evaluar diferentes aspectos del autoconcepto en niños, adolescentes y adultos, que incluyen las dimensiones social, académico/profesional, emocional, familiar y física (García \& Musitu, 2014). En cuanto al análisis de las propiedades psicométricas del instrumento, se señala que el cuestionario AF5 ofrece índices de fiabilidad superiores a 0,71 (Esnaola, Rodríguez \& Goñi, 2011).

\subsection{Procedimiento}

Recolección de información: Los estudiantes de los grupos que quedaron en la muestra diligenciaron el instrumento AF5 bajo la supervisión de las docentes investigadoras, y firmaron el consentimiento informado. Variables utilizadas en la investigación: Programa, semestre, género, puntajes académico-laboral, social, emocional, familiar y físico.

\subsection{Análisis de datos}

Con base en la información disponible, se construyó una matriz de datos a la que se le realizó el siguiente análisis estadístico:

2.5.1. Comparación estadística de medias de autoconceptos entre programas, para un mismo semestre, y un mismo género.

Se utilizó la prueba de Shapiro-Wilk (Díaz, 2009) para determinar la normalidad de los datos, si estos pasaban tal prueba, se establece la homogeneidad de varianzas mediante la dócima de Bartlett (Walpole, Myers, Myers \& Ye, 2012); posteriormente se realizó Anova a una vía para comparar entre medias. En caso que no se pasara la prueba de normalidad o de varianzas homogéneas, se realizaba la prueba no paramétrica de Kruskal-Wallis (Sheskin, 2007).

2.5.2. Comparación entre los resultados promedio del autoconcepto académico-laboral, social, emocional, familiar y físico, entre hombres de primeros y últimos semestres y entre mujeres de primeros y últimos semestres.

En este caso si los autoconceptos pasaban la prueba de normalidad, se realizaba una prueba f para determinar si las varianzas eran iguales entre ambos grupos, posteriormente se aplicó la prueba $t$ 
correspondiente. En caso que no se pasara la prueba de normalidad, se realizaba la prueba no paramétrica U de Mann-Whitney (Sheskin, 2007).

2.5.3. Comparación entre los resultados promedio obtenidos para cada autoconcepto en cada semestre y por cada género, contra el correspondiente del baremo.

Utilizando la misma metodología del enunciado anterior.

\section{RESULTADOS}

3.1. Comparación estadística de medias de autoconceptos entre programas, para un mismo semestre y un mismo género.

Las Tablas 2, 3 y 4 muestran el Pvalor del Anova o la prueba de Kruskal-Wallis correspondiente, cuando se compararon entre programas, los hombres de primeros y últimos semestres, así como las mujeres de primeros y últimos semestres. Únicamente se muestran aquellos programas estadísticamente diferentes (tercera columna) y se analiza en la cuarta columna en qué radica tal diferencia.

\subsubsection{Autoconcepto académico-laboral.}

Los hombres de primeros y últimos semestres del programa de Licenciatura en Filosofía y Letras y los estudiantes de Historia y Licenciatura en Ciencias Sociales presentan menores promedios en este autoconcepto que sus compañeros de Medicina y de Licenciatura en Educación Fí- sica. Igual ocurre con las mujeres de primeros semestres de Licenciatura en Filosofía y Letras y las de Historia y Lic. en Ciencias Sociales con respecto a las estudiantes de Desarrollo Familiar (Tabla 2). Para las estudiantes de últimos semestres no se encontró diferencia significativa entre programas para este autoconcepto (Pvalor $=0,4442$ ).

Tabla 2.

Comparación estadística de medias por programas para el autoconcepto académico laboral

\begin{tabular}{|c|c|c|c|}
\hline Se comparan & Pvalor & Programas estadísticamente diferentes & Conclusión \\
\hline \multirow[t]{4}{*}{$\begin{array}{l}\text { Hombres de } \\
\text { primeros } \\
\text { semestres }\end{array}$} & \multirow[t]{4}{*}{0,0026} & $\begin{array}{l}\text { Lic. Educación física vs. Lic. Filosofía y } \\
\text { letras }\end{array}$ & $\begin{array}{l}\text { Los estudiantes del programa de Licenciatura en filosofía } \\
\text { y letras obtienen promedios inferiores a los obtenidos } \\
\text { por los de la Licenciatura en educación física }\end{array}$ \\
\hline & & Medicina vs. Lic. Filosofía y letras & $\begin{array}{l}\text { Los estudiantes del programa de Licenciatura en filosofía } \\
\text { y letras obtienen promedios inferiores a los obtenidos } \\
\text { por los de Medicina }\end{array}$ \\
\hline & & $\begin{array}{l}\text { Lic. Educación física vs. Historia y Lic. } \\
\text { Ciencias sociales }\end{array}$ & $\begin{array}{l}\text { Los estudiantes de los programas de Historia y Lic. } \\
\text { Ciencias sociales obtienen promedios inferiores a los } \\
\text { obtenidos por los de la Licenciatura en educación física }\end{array}$ \\
\hline & & $\begin{array}{c}\text { Medicina vs. Historia y Lic. Ciencias } \\
\text { sociales }\end{array}$ & $\begin{array}{l}\text { Los estudiantes de los programas de Historia y Lic. } \\
\text { Ciencias sociales obtienen promedios inferiores a los } \\
\text { obtenidos por los de Medicina }\end{array}$ \\
\hline \multirow[t]{2}{*}{$\begin{array}{l}\text { Hombres de } \\
\text { últimos } \\
\text { semestres }\end{array}$} & \multirow[t]{2}{*}{0,0003} & $\begin{array}{c}\text { Lic. Educación física vs. Lic. Filosofía y } \\
\text { letras }\end{array}$ & $\begin{array}{l}\text { Los estudiantes del programa de Licenciatura en filosofía } \\
\text { y letras obtienen promedios inferiores a los obtenidos } \\
\text { por los de la Licenciatura en educación física }\end{array}$ \\
\hline & & Medicina vs. Lic. Filosofía y letras & $\begin{array}{l}\text { Los estudiantes del programa de Licenciatura en filosofía } \\
\text { y letras obtienen promedios inferiores a los obtenidos } \\
\text { por los de Medicina }\end{array}$ \\
\hline
\end{tabular}




\begin{tabular}{|c|c|c|c|}
\hline & \multirow{4}{*}{0,0129} & $\begin{array}{l}\text { Lic. Educación física vs. Historia y Lic. } \\
\text { Ciencias sociales }\end{array}$ & $\begin{array}{l}\text { Los estudiantes de los programas de Historia y Lic. } \\
\text { Ciencias sociales obtienen promedios inferiores a los } \\
\text { obtenidos por los de la Licenciatura en educación física }\end{array}$ \\
\hline \multirow{3}{*}{$\begin{array}{l}\text { Mujeres de } \\
\text { primeros } \\
\text { semestres }\end{array}$} & & $\begin{array}{c}\text { Medicina vs. Historia y Lic. Ciencias } \\
\text { sociales }\end{array}$ & $\begin{array}{l}\text { Los estudiantes de los programas de Historia y Lic. } \\
\text { Ciencias sociales obtienen promedios inferiores a los } \\
\text { obtenidos por los de Medicina }\end{array}$ \\
\hline & & $\begin{array}{c}\text { Desarrollo familiar vs. Lic. Filosofía y } \\
\text { letras }\end{array}$ & $\begin{array}{c}\text { Las estudiantes del programa de Lic. En Filosofía y letras } \\
\text { obtienen promedios inferiores a los obtenidos por las de } \\
\text { Desarrollo familiar }\end{array}$ \\
\hline & & $\begin{array}{l}\text { Desarrollo familiar vs. Historia y Lic. } \\
\text { Ciencias Sociales }\end{array}$ & $\begin{array}{l}\text { Las estudiantes de los programas de Historia y Lic. } \\
\text { Ciencias Sociales obtienen promedios inferiores a los } \\
\text { obtenidos por las de Desarrollo familiar }\end{array}$ \\
\hline
\end{tabular}

\subsubsection{Autoconcepto social.}

La Tabla 3 muestra que los estudiantes hombres de primeros semestres del programa de Licenciatura en Filosofía y Letras presentan menores promedios en el autoconcepto social que sus compañeros de los programas de Licenciatura en Educación Física y de Historia y Licenciatura en Ciencias Sociales. En cuanto a los estudiantes hombres de últimos semestres, nuevamente el programa de Licenciatura en Filosofía y Letras, presentan menores medias en este autoconcepto que los estudiantes de la Licenciatura en Educación Física y de Medicina. Para las estudiantes de primeros y últimos semestres no se encontró diferencia significativa entre programas para este autoconcepto (Pvalores de 0,4587 y 0,9898, respectivamente).

Tabla 3.

Comparación estadística de medias por programas para el autoconcepto social

\begin{tabular}{|c|c|c|c|}
\hline Se comparan & Pvalor & Programas estadísticamente diferentes & Conclusión \\
\hline \multirow[t]{2}{*}{$\begin{array}{l}\text { Hombres de } \\
\text { primeros } \\
\text { semestres }\end{array}$} & \multirow[t]{2}{*}{0,0138} & $\begin{array}{l}\text { Lic. Educación física vs. Lic. Filosofía y } \\
\text { letras }\end{array}$ & $\begin{array}{c}\text { Los estudiantes del programa de Licenciatura en } \\
\text { filosofía y letras obtienen promedios inferiores a } \\
\text { los obtenidos por los de la Licenciatura en } \\
\text { educación física }\end{array}$ \\
\hline & & $\begin{array}{c}\text { Historia y Lic. Ciencias sociales vs. Lic. } \\
\text { Filosofía y letras }\end{array}$ & $\begin{array}{c}\text { Los estudiantes del programa de Licenciatura en } \\
\text { filosofía y letras obtienen promedios inferiores a } \\
\text { los obtenidos por los de Historia y Lic. En } \\
\text { Ciencias sociales }\end{array}$ \\
\hline \multirow[t]{2}{*}{$\begin{array}{l}\text { Hombres de } \\
\text { últimos semestres }\end{array}$} & \multirow[t]{2}{*}{0,0451} & $\begin{array}{c}\text { Lic. Educación física vs. Lic. Filosofía y } \\
\text { letras }\end{array}$ & $\begin{array}{c}\text { Los estudiantes del programa de Licenciatura en } \\
\text { filosofía y letras obtienen promedios inferiores a } \\
\text { los obtenidos por los de la Licenciatura en } \\
\text { educación física }\end{array}$ \\
\hline & & Medicina vs. Lic. Filosofía y letras & $\begin{array}{l}\text { Los estudiantes del programa de Licenciatura en } \\
\text { filosofía y letras obtienen promedios inferiores a } \\
\text { los obtenidos por los de Medicina }\end{array}$ \\
\hline
\end{tabular}

\subsubsection{Autoconcepto emociona.}

Únicamente se muestran diferencias entre programas para este autoconcepto, para los estudiantes hombres de primeros semestres (Tabla 4), siendo los del programa de Licenciatura en Filosofía y Letras, quienes presentan las mayores medias cuando se comparan con los demás programas. Para los estudiantes hombres de últimos semestres y mujeres de primeros y últimos semestres, no se 
encontró diferencia significativa en este autoconcepto (Pvalores de 0,7831, 0,6688 y 0,9153, respectivamente).

Tabla 4.

Comparación estadística de medias por programas para el autoconcepto emocional

\begin{tabular}{|c|c|c|c|}
\hline Se comparan & Pvalor & Programas estadísticamente diferentes & Conclusión \\
\hline \multirow[t]{3}{*}{$\begin{array}{l}\text { Hombres de } \\
\text { primeros } \\
\text { semestres }\end{array}$} & \multirow[t]{3}{*}{0,0131} & $\begin{array}{c}\text { Lic. Educación física vs. Lic. Filosofía y } \\
\text { letras }\end{array}$ & $\begin{array}{l}\text { Los estudiantes del programa de Licenciatura en } \\
\text { educación física, obtienen promedios inferiores a los } \\
\text { obtenidos por los de } \\
\text { Licenciatura en filosofía y letras }\end{array}$ \\
\hline & & $\begin{array}{c}\text { Historia y Lic. Ciencias sociales vs. Lic. } \\
\text { Filosofía y letras }\end{array}$ & $\begin{array}{l}\text { Los estudiantes de los programas de Historia y de } \\
\text { Licenciatura Ciencias sociales, obtienen promedios } \\
\text { inferiores a los obtenidos por los de } \\
\text { Licenciatura en filosofía y letras }\end{array}$ \\
\hline & & Medicina vs. Lic. Filosofía y letras & $\begin{array}{l}\text { Los estudiantes del programa de Medicina, obtienen } \\
\text { promedios inferiores a los obtenidos por los de } \\
\text { Licenciatura en filosofía y letras }\end{array}$ \\
\hline
\end{tabular}

\subsubsection{Autoconcepto familiar.}

Para este autoconcepto no se apreciaron diferencias significativas entre programas (Pvalores de $0,0519,0,1166,0,2636$ y 0,5338 , respectivamente).

\subsubsection{Autoconcepto físico.}

Los estudiantes hombres de primeros y últimos semestres de Licenciatura en Educación Física obtienen los mayores puntajes cuando se comparan con los estudiantes de Historia, Licenciatura en Ciencias sociales, Medicina y Licenciatura en Filosofía y Letras; siendo estos últimos quienes muestran las menores medias; así mismo, las medias de Historia y Lic. Ciencias Sociales y de Medicina en lo físico son estadísticamente iguales (Tabla 5). En cuanto a las mujeres, no se encontró diferencia estadística entre programas (Pvalores de 0,8843 y 0,1350, respectivamente).

Tabla 5.

Comparación estadística de medias por programas para el autoconcepto físico

\begin{tabular}{|c|c|c|c|}
\hline Se comparan & Pvalor & Programas estadísticamente diferentes & Conclusión \\
\hline \multirow[t]{5}{*}{$\begin{array}{l}\text { Hombres de } \\
\text { primeros } \\
\text { semestres }\end{array}$} & \multirow[t]{5}{*}{0,0000} & $\begin{array}{l}\text { Lic. Educación física vs. Lic. Filosofía y } \\
\text { letras }\end{array}$ & $\begin{array}{l}\text { Los estudiantes del programa de Licenciatura en } \\
\text { filosofía y letras, obtienen promedios inferiores a los } \\
\text { obtenidos por los de Licenciatura en educación física }\end{array}$ \\
\hline & & $\begin{array}{c}\text { Historia y Lic. Ciencias sociales vs. Lic. } \\
\text { Filosofía y letras }\end{array}$ & $\begin{array}{c}\text { Los estudiantes del programa de Licenciatura en } \\
\text { filosofía y letras, obtienen promedios inferiores a los } \\
\text { obtenidos por los de Historia y de Licenciatura en } \\
\text { Ciencias sociales }\end{array}$ \\
\hline & & Medicina vs. Lic. Filosofía y letras & $\begin{array}{c}\text { Los estudiantes del programa de Licenciatura en } \\
\text { filosofía y letras, obtienen promedios inferiores a los } \\
\text { obtenidos por los de Medicina }\end{array}$ \\
\hline & & $\begin{array}{l}\text { Lic. Educación física vs. Historia y Lic. } \\
\text { Ciencias sociales }\end{array}$ & $\begin{array}{l}\text { Los estudiantes de los programas de Historia y Lic. } \\
\text { Ciencias sociales, obtienen promedios inferiores a los } \\
\text { obtenidos por los de Licenciatura en educación física }\end{array}$ \\
\hline & & Lic. Educación física vs. Medicina & $\begin{array}{l}\text { Los estudiantes del programa de Medicina, obtienen } \\
\text { promedios inferiores a los obtenidos por los de } \\
\text { Licenciatura en educación física }\end{array}$ \\
\hline
\end{tabular}




\begin{tabular}{|c|c|c|c|}
\hline \multirow[t]{5}{*}{$\begin{array}{l}\text { Hombres de } \\
\text { primeros } \\
\text { semestres }\end{array}$} & \multirow[t]{5}{*}{0,0000} & $\begin{array}{l}\text { Lic. Educación física vs. Lic. Filosofía y } \\
\text { letras }\end{array}$ & $\begin{array}{l}\text { Los estudiantes del programa de Licenciatura en } \\
\text { filosofía y letras, obtienen promedios inferiores a los } \\
\text { obtenidos por los de Licenciatura en educación física }\end{array}$ \\
\hline & & $\begin{array}{l}\text { Historia y Lic. Ciencias sociales vs. Lic. } \\
\text { Filosofía y letras }\end{array}$ & $\begin{array}{c}\text { Los estudiantes del programa de Licenciatura en } \\
\text { filosofía y letras, obtienen promedios inferiores a los } \\
\text { obtenidos por los de Historia y de Licenciatura en } \\
\text { Ciencias sociales }\end{array}$ \\
\hline & & Medicina vs. Lic. Filosofía y letras & $\begin{array}{c}\text { Los estudiantes del programa de Licenciatura en } \\
\text { filosofía y letras, obtienen promedios inferiores a los } \\
\text { obtenidos por los de Medicina }\end{array}$ \\
\hline & & $\begin{array}{l}\text { Lic. Educación física vs. Historia y Lic. } \\
\text { Ciencias sociales }\end{array}$ & $\begin{array}{l}\text { Los estudiantes de los programas de Historia y Lic. } \\
\text { Ciencias sociales, obtienen promedios inferiores a los } \\
\text { obtenidos por los de Licenciatura en educación física }\end{array}$ \\
\hline & & Lic. Educación física vs. Medicina & $\begin{array}{l}\text { Los estudiantes del programa de Medicina, obtienen } \\
\text { promedios inferiores a los obtenidos por los de } \\
\text { Licenciatura en educación física }\end{array}$ \\
\hline
\end{tabular}

\subsection{Comparación estadística de medias de autoconceptos entre semestres, para un mismo} programa y un mismo género.

La Tabla 6 muestra el Pvalor de la prueba t o de la U de Mann-Whitney, encontrado al comparar para un mismo programa y un mismo género, cada uno de los autoconceptos (únicamente se muestran aquellos casos para los cuales se detectó diferencia estadística). Es claro que en todos los programas donde se evaluaron mujeres excepto en la Licenciatura en Filosofía y Letras (Historia y Licenciatura en Ciencias Sociales, Medicina, Desarrollo Familiar y Psicología), el autoconcepto académico-laboral promedio se incrementa cuando las estudiantes adelantan en su programa académico.

Para los hombres solo se observaron incrementos en el autoconcepto académico laboral en el programa de Medicina y en el físico para la Licenciatura en Educación Física, pero llama la atención que cuando los estudiantes de la licenciatura en Filosofía y Letras avanzan en su programa, disminuya su calificación promedio en lo emocional.

Tabla 6.

Pvalor de la Prueba U de Mann-Whitney o t, al comparar las medias entre semestres para un mismo programa y un mismo género

\begin{tabular}{|c|c|c|c|c|}
\hline Programa & Autoconcepto & Estudiantes comparados & Pvalor & Conclusión \\
\hline $\begin{array}{l}\text { Historia y Lic. } \\
\text { Ciencias sociales }\end{array}$ & $\begin{array}{l}\text { Académico } \\
\text { laboral }\end{array}$ & $\begin{array}{l}\text { Mujeres primeros semestres vs } \\
\text { Mujeres últimos semestres }\end{array}$ & 0,03504 & $\begin{array}{l}\text { Las mujeres de últimos semestres de } \\
\text { Historia y Lic. En Ciencias sociales, } \\
\text { obtienen mayores promedios en lo } \\
\text { académico laboral, que las de primeros } \\
\text { semestres }\end{array}$ \\
\hline Medicina & $\begin{array}{l}\text { Académico } \\
\text { laboral }\end{array}$ & $\begin{array}{l}\text { Hombres primeros semestres } \\
\text { vs Hombres últimos semestres }\end{array}$ & 0,00905 & $\begin{array}{l}\text { Los hombres de últimos semestres de } \\
\text { Medicina, obtienen mayores } \\
\text { promedios en lo académico laboral, } \\
\text { que los de primeros semestres }\end{array}$ \\
\hline Medicina & $\begin{array}{l}\text { Académico } \\
\text { laboral }\end{array}$ & $\begin{array}{c}\text { Mujeres primeros semestres vs } \\
\text { Mujeres últimos semestres }\end{array}$ & 0,01426 & $\begin{array}{l}\text { Las mujeres de últimos semestres de } \\
\text { Medicina, obtienen mayores } \\
\text { promedios en lo académico laboral, } \\
\text { que las de primeros semestres }\end{array}$ \\
\hline $\begin{array}{l}\text { Desarrollo } \\
\text { familiar* }\end{array}$ & $\begin{array}{l}\text { Académico } \\
\text { laboral }\end{array}$ & $\begin{array}{l}\text { Mujeres primeros semestres vs } \\
\text { Mujeres últimos semestres }\end{array}$ & 0,03257 & $\begin{array}{l}\text { Las estudiantes de últimos semestres } \\
\text { de Desarrollo familiar, obtienen }\end{array}$ \\
\hline
\end{tabular}


Diana Marcela Montoya Londoño, Victoria Eugenia Pinilla Sepúlveda, Carmen Dussán Luberth

\begin{tabular}{|c|c|c|c|c|}
\hline & & & & $\begin{array}{l}\text { mayores promedios en lo académico } \\
\text { laboral, que las de primeros semestres }\end{array}$ \\
\hline Psicología* & $\begin{array}{l}\text { Académico } \\
\text { laboral }\end{array}$ & $\begin{array}{l}\text { Mujeres primeros semestres vs } \\
\text { Mujeres últimos semestres }\end{array}$ & 0,02259 & $\begin{array}{l}\text { Las estudiantes de últimos semestres } \\
\text { de Psicología, obtienen mayores } \\
\text { promedios en lo académico laboral, } \\
\text { que las de primeros semestres }\end{array}$ \\
\hline Filosofía & Emocional & $\begin{array}{l}\text { Hombres primeros semestres } \\
\text { vs Hombres últimos semestres }\end{array}$ & 0,00234 & $\begin{array}{l}\text { Los hombres de últimos semestres de } \\
\text { Lic. En Filosofía y letras, obtienen } \\
\text { menores promedios en lo emocional, } \\
\text { que los de primeros semestres }\end{array}$ \\
\hline $\begin{array}{l}\text { Licenciatura en } \\
\text { educación física** }\end{array}$ & Físico & $\begin{array}{c}\text { Primeros semestres vs Últimos } \\
\text { semestres }\end{array}$ & 0,03743 & $\begin{array}{l}\text { Los hombres de últimos semestres de } \\
\text { Licenciatura en educación física, } \\
\text { obtienen mayores promedios en lo } \\
\text { físico, que los de primeros semestres }\end{array}$ \\
\hline
\end{tabular}

3.3. Comparación estadística de medias de autoconceptos contra el baremo correspondiente, entre semestres para un mismo género y un mismo programa.

Al comparar las medias obtenidas por los estudiantes en cada uno de los autoconceptos, contra el baremo correspondiente, se encontró que los estudiantes de los programas de Ciencias Sociales y de Historia de la Universidad de Caldas, en promedio obtienen calificaciones superiores a las del baremo así: hombres en lo académico-laboral, familiar y físico y mujeres en lo académicolaboral, emocional y físico (Tabla 7).

Así mismo se observó que las estudiantes de Desarrollo Familiar, en promedio, obtienen mayores calificaciones que las reportadas por el baremo en lo académico-laboral, social, familiar y físico (Tabla 8) y las de Psicología en los autoconceptos académico-laboral, social, emocional y físico (Tabla 9).

Para el caso del programa de Medicina, los hombres y mujeres obtienen mayores medias que la reportada por el baremo en lo académico-laboral, familiar y físico; además de los hombres en lo social (Tabla 10).

Tabla 7.

Pvalores de las pruebas al comparar las medias obtenidas contra el baremo para los estudiantes de los programas de Historia y Licenciatura en Ciencias sociales

\begin{tabular}{|c|c|c|c|c|c|}
\hline Individuos comparados & $\begin{array}{c}\text { Académico } \\
\text { Laboral }\end{array}$ & Social & Emocional & Familiar & Físico \\
\hline $\begin{array}{c}\text { Hombres primeros semestres vs. } \\
\text { Hombres baremo }\end{array}$ & 0,02593 & 0,17398 & 0,88743 & 0,00284 & 0,00008 \\
\hline $\begin{array}{c}\text { Hombres últimos semestres vs. } \\
\text { Hombres baremo }\end{array}$ & 0,00292 & 0,61309 & 0,38273 & 0,05179 & 0,00099 \\
\hline $\begin{array}{c}\text { Mujeres primeros semestres vs. } \\
\text { Mujeres baremo }\end{array}$ & 0,44441 & 0,63403 & 0,04777 & 0,77706 & 0,00002 \\
\hline $\begin{array}{l}\text { Mujeres últimos semestres vs. } \\
\text { Mujeres baremo }\end{array}$ & 0,00082 & 0,15710 & 0,58854 & 0,19940 & 0,00031 \\
\hline
\end{tabular}


Tabla 8.

Pvalores de las pruebas al comparar las medias obtenidas contra el baremo para las estudiantes del programa de Desarrollo familiar

\begin{tabular}{cccccc}
\hline $\begin{array}{c}\text { Estudiantes comparados contra } \\
\text { el baremo }\end{array}$ & Académico-Laboral & Social & Emocional & Familiar & Físico \\
\hline Primeros semestres & 0,00000 & 0,55862 & 0,71011 & 0,01357 & 0,00000 \\
Últimos semestres & 0,00000 & 0,00809 & 0,39198 & 0,02617 & 0,00000 \\
\hline
\end{tabular}

Tabla 9.

Pvalores de las pruebas al comparar las medias obtenidas contra el baremo para las estudiantes del programa de Psicología

\begin{tabular}{cccccc}
\hline $\begin{array}{c}\text { Estudiantes comparados contra } \\
\text { el baremo }\end{array}$ & Académico-Laboral & Social & Emocional & Familiar & Físico \\
\hline Primeros semestres & 0,00538 & 0,99577 & 0,03334 & 0,46407 & 0,00001 \\
Últimos semestres & 0,00000 & 0,04724 & 0,90649 & 0,50667 & 0,00001 \\
\hline
\end{tabular}

Tabla 10.

Pvalores de las pruebas al comparar las medias obtenidas contra el baremo para los estudiantes del programa de Medicina

\begin{tabular}{|c|c|c|c|c|c|}
\hline Individuos comparados & $\begin{array}{l}\text { Académico- } \\
\text { Laboral }\end{array}$ & Social & Emocional & Familiar & Físico \\
\hline $\begin{array}{l}\text { Hombres primeros semestres vs. } \\
\text { Hombres baremo }\end{array}$ & 0,00000 & 0,44943 & 0,23102 & 0,00004 & 0,00000 \\
\hline $\begin{array}{c}\text { Hombres últimos semestres vs. } \\
\text { Hombres baremo }\end{array}$ & 0,00000 & 0,01474 & 0,84755 & 0,00060 & 0,00000 \\
\hline $\begin{array}{c}\text { Mujeres primeros semestres vs. } \\
\text { Mujeres baremo }\end{array}$ & 0,00958 & 0,44321 & 0,79043 & 0,01796 & 0,00001 \\
\hline $\begin{array}{c}\text { Mujeres últimos semestres vs. } \\
\text { Mujeres baremo }\end{array}$ & 0,00001 & 0,09688 & 0,50516 & 0,00032 & 0,00032 \\
\hline
\end{tabular}

Los estudiantes de Licenciatura en educación física, en promedio presentan mayores medias que la del baremo correspondiente, en lo académico-laboral, social, familiar y físico (tabla 11).

Tabla 11.

Pvalores de las pruebas al comparar las medias obtenidas contra el baremo para los estudiantes del programa de Licenciatura en educación física

\begin{tabular}{cccccc}
\hline $\begin{array}{c}\text { Individuos comparados contra el } \\
\text { baremo }\end{array}$ & $\begin{array}{c}\text { Académico- } \\
\text { Laboral }\end{array}$ & Social & Emocional & Familiar & Físico \\
\hline Primeros semestres & 0,00000 & 0,00055 & 0,14484 & 0,00171 & 0,00000 \\
Últimos semestres & 0,00000 & 0,01194 & 0,73233 & 0,00019 & 0,00000 \\
\hline
\end{tabular}

Mientras que para las medias de los autoconceptos de los estudiantes del programa de Licenciatura en Filosofía y Letras, cuando se comparan con las del baremo correspondiente, se observa que tales promedios son superiores al del baremo para los hombres de primeros semestres en lo emocional 
y las mujeres de primeros semestres en lo físico, así como para las de últimos semestres en lo físico y lo académico laboral (tabla 12).

Tabla 12.

Pvalores de las pruebas al comparar las medias obtenidas contra el baremo para los estudiantes del programa de Licenciatura en Filosofía y letras

\begin{tabular}{cccccc}
\hline \multicolumn{1}{c}{ Individuos comparados } & $\begin{array}{c}\text { Académico } \\
\text { Laboral }\end{array}$ & Social & Emocional & Familiar & Físico \\
\hline $\begin{array}{c}\text { Hombres primeros semestres vs. Hombres } \\
\text { baremo }\end{array}$ & 0,46933 & 0,11430 & 0,00000 & 0,86343 & 0,43098 \\
Hombres últimos semestres vs. Hombres baremo & 0,94908 & 0,12938 & 0,90457 & 0,47809 & 0,70284 \\
Mujeres primeros semestres vs. Mujeres baremo & 0,68359 & 0,09447 & 0,61976 & 0,70175 & 0,00769 \\
Mujeres últimos semestres vs. Mujeres baremo & 0,02823 & 0,25624 & 0,13113 & 0,16852 & $\mathbf{0 , 0 0 0 0 2}$ \\
\hline
\end{tabular}

La diferencia mostrada en las tablas 7 a 12 en lo que respecta al baremo, sugiere la necesidad de estandarizar esta prueba los jóvenes universitarios del contexto colombiano.

\section{DISCUSIÓN}

El autoconcepto como categoría de la Psicología, se ha considerado esencial en el desarrollo de la personalidad y en el bienestar psicosocial del individuo, en cuanto se reconoce su importancia en el estado emocional y en el desarrollo social de la persona, así como en la consolidación de su identidad y sentido de satisfacción personal. El autoconcepto positivo está en la base del buen funcionamiento personal, social y profesional, dependiendo de él, en buena medida el sentirse bien consigo mismo (Goñi-Palacios, 2009; Guitron, 2013; Bustos, Oliver \& Galiana, 2015). El autoconcepto puede definirse así, como las concepciones y representaciones mentales que una persona tiene de sí misma, en tal sentido, se entiende como la percepción y valoración que una persona hace de sí misma en tres dimensiones básicas: cognitiva, afectiva y comportamental (Burns, 1990; Salvador-Ferrer, 2012).

La concepción multidimensional y jerárquica del autoconcepto, representada por la estructura propuesta por Shavelson, Hubner y Stanton (1976), constituye el planteamiento actualmente másrespaldado por la comunidad científica en lo que a la autopercepción se refiere (RevueltaRevuelta, Rodríguez-Fernández, Ruiz de Azúa-Ormaza, \& Ramos-Díaz, 2015). De hecho, puede indicarse que a partir de los diferentes modelos teóricos desde los cuales se ha estudiado el autoconcepto (nomotético, jerárquico, taxonómico y compensatorio) (Byrne, 1984; Strein, 1993), puede considerarse que el modelo que ha demostrado más fundamentación empírica en estudios transversales y longitudinales, es el jerárquico de Shavelson, Hubner \& Stanton (1976) (Fleming \& Watts, 1980; Fleming \& Courteney, 1984; Byrne \& Shavelson, 1986; Byrne, 1986; Marsh, Byrne, \& Shavelson, 1988; Marsh, Craven y Debus, 1991; González-Pienda, Nuñez-Pérez, Valle-Arias, 1992; Marsh, \& Ayotte, 2003; Marsh, 2005; Marsh y Craven, 2006; Moritz, Read, Clark, Callahan y 
Albaugh, 2009; Véliz - Burgos, \&, Apodaca- Urquijo, 2012a), modelo desde el cual se reconoce el autoconcepto en perspectiva multidimensional.

En esta perspectiva multidimensional, los autores conciben el autoconcepto como la percepción que el individuo tiene sobre sí mismo, basada directamente en sus experiencias con los demás y en las atribuciones que él mismo hace de su conducta (Polo-Sánchez \& López-Justicia, 2012;Elexpuru, Villardón \& Yániz, 2013; Silva-Ramos, 2016). Desde el modelo jerárquico de Shavelson, Hubner \& Stanton (1976), el autoconcepto no puede ser descrito desde puntuaciones homogéneas en un mismo individuo, por el contrario este tiene una estructura jerarquizada en la que sus dimensiones tienen diferentes significados e importancia en función de los valores y la edad de los sujetos. Se considera que el concepto, que el individuo tiene de sí mismo, es experimental y tiene un carácter evaluativo, por lo mismo, se va construyendo y diferenciando a lo largo del ciclo vital del individuo, a partir de sus interacciones sociales y proceso educacional (Garaigordobil, Aliri, \& Fontaneda, 2009b; Véliz, 2010; García, \& Gracia, 2010; Valenzuela-Zambrano \& López-Justicia, 2015).

En el modelo jerárquico de Shavelson, Hubner \& Stanton (1976) se asume que en la parte más elevada de la pirámide se encuentra la concepción global del autoconcepto que tiene el individuo (autoconcepto general/ AG), en la parte central se ubica el autoconcepto académico (AA/ Matemático, verbal) y no académico,(A- NA/Físico, emocional y social) y en la base de la estructura estarían ubicadas las dimensiones particulares (Mestre \& Pérez-Delgado, 1994; Esteve, 2005; Cazalla-Luna \& Morelo, 2013; Fernández-Zabala, Goñi, Rodríguez-Fernández \& Goñi, 2015). Así entendido el autoconcepto constituye el concepto que el individuo tiene acerca de sí mismo y está conformado por la suma de pensamientos y sentimientos acerca de quién es, cómo se siente y qué hace.

Desde el contexto de la formación profesional del individuo, parecen evidenciarse ciertos perfiles en torno a las posibilidades de desarrollo del autoconcepto, que se van construyendo al principio de la carrera, así como hacia el final de la formación en pregrado, entendido así que la formación en la Educación superior, implica más que sólo el aprendizaje de una disciplina, la generación de aptitudes emocionales personales y sociales, que garanticen la permanencia y graduación, entre las cuales el desarrollo de un sano autoconcepto parecería ser un propósito fundamental en la formación y el rendimiento académico, en cuanto permitiría mejorar los indicadores críticos de deserción, repetición de curso y la experiencia de aprendizaje en el pregrado, así como el desarrollo de acciones desde los programas de formación en el pregrado que permitan apoyar el proceso de la vida universitaria y favorecer el éxito académico. En tal sentido, puede señalarse que autores como Musitu, Garcia \& Gutiérrez (1997), confirmaron la importancia que tiene el autoconcepto sobre el rendimiento académico, al demostrar que la percepción y los sentimientos al respecto de si mismo son factores determinantes en el éxito académico de los individuos (Urquijo, 2002), en cuanto consideran que un autoconcepto negativo, genera falta de confianza en sí mismo, una visión distorsionada de sí, sentimientos de minusvalía e incapacidad, lo que puede reflejarse posteriormente, en bajo rendimiento académico y social (Parra, Restrepo, Usuga, Castañeda, Estrada, Uñates, Gil, \& Mendoza, 2015). 
La importancia del autoconcepto en la formación universitaria, radica en su reconocida contribución a la formación de la personalidad y en su influencia en el rendimiento académico, en cuanto tiene que ver con la competencia social, ya que influye sobre la persona en cómo siente, cómo piensa, cómo aprende, cómo se valora, cómo se relaciona con los demás y en cómo se comporta (Clark, Clemes \& Bean, 2000; Peralta - Sánchez \& Sánchez - Roda, 2003; Costa \& Tabernero, 2012 ), de ahí que se reconozca que las experiencias académicas de éxito o fracaso inciden significativamente sobre el autoconcepto del estudiante, lo cual podría ser explicado mediante el papel de las evaluaciones de los otros significativos (Tajfel \& Turner, 1986; Peralta-Sánchez \& Sánchez-Roda, 2003; Costa \& Tabernero, 2012; Cordero-Tapia \& Duran, 2015).

Entre las diferencias encontradas a nivel de los programas de pregrado, en relación con el desempeño en los diferentes factores del autoconcepto que se evaluaron, puede decirse que:

El autoconcepto académico/laboral se refiere a la percepción que el sujeto tiene de la calidad del desempeño de su rol como estudiante o trabajador. Un autoconcepto académico alto correlaciona con alto rendimiento académico y satisfacción en el estudio. Desde la aplicación y desempeño en la prueba AF5 (García \& Músitu, 2009, García \& Músitu, 2014) puede decirse que la dimensión académico-laboral gira en torno a dos ejes: el primero se refiere al sentimiento que el estudiante tiene del desempeño de su rol a través de sus profesores (ser buen estudiante, etc), y el segundo a cualidades específicas valoradas en este contexto (ser inteligente, etc). A nivel del autoconcepto académico/ laboral, los hombres de primeros y últimos semestres de los programas de pregrado en Filosofía, Historia, Licenciatura en Ciencias Sociales obtuvieron menores promedios que los estudiantes de Medicina y Licenciatura en educación física. Se encontró que las mujeres de primeros semestres de los programas de pregrado en Filosofía, Historia, y Licenciatura en Ciencias Sociales obtuvieron menores promedios que las estudiantes de Desarrollo Familiar. A nivel de las estudiantes de últimos semestres no se encontraron diferencias significativas.

Los hallazgos encontrados permiten suponer un posible efecto de la disciplina en el factor del autoconcepto académico/laboral, en cuanto se establecen menores promedios entre estudiantes de primeros y últimos semestres de unas carreras, respecto a otras. Es tal sentido, parecería ser que desde la formación académica impartida en las carreras de Medicina y Licenciatura en educación física para el caso de los hombres y que el programa de Desarrollo familiar en el caso de las mujeres, se orientará un proceso de formación que más allá del solo estudio de la disciplina, trabajará otro tipo de variables relacionadas con la vocación, identidad profesional y reconocimiento del estatus de la carrera, en cuanto se ha considerado que mejores desempeños a nivel del autococepto académico / laboral correlacionan con el ajuste psicosocial, la percepción del rendimiento académico/laboral, la calidad en la ejecución del trabajo, la aceptación y estima de los compañeros, el liderazgo y la responsabilidad (García \& Músitu, 2009; Esnaola, Goñi \& Madariaga, 2008; Elexpuru, Villardón, \& Yániz, 2013, García \& Músitu, 2014).

Estos hallazgos coinciden con algunas investigaciones en las que se ha encontrado que una percepción positiva de sí mismo, junto con la suma de habilidades que posee el sujeto, son fundamentales para el desempeño escolar y por lo tanto, para la consecución de los logros, en cuanto se considera que el aprendizaje aumenta cuando el sujeto confía en sus propias capacidades 
y se siente autoeficaz, responsable y gestor de su propio proceso, percepción que es consolidada desde sus interacciones sociales, para este caso, interacciones escolares (Acosta, 1988; Machargo, 1989; González \& Tourón, 1992; Amezcua \& Pichardo, 2000; Vicuña, 2004;García, 2004; Avendaño, Jiménez \& Senior, 2008; Ramírez, 2012; García-Fernández, Inglés, Díaz-Herrero, Lagos-Sanmartín, Torregrosa \& Gonzálvez, 2016).

El autoconcepto social, es entendido como la percepción que tiene el sujeto de su desempeño en las relaciones sociales. Se considera que correlaciona positivamente con el ajuste y el bienestar psicosocial, el rendimiento académico y laboral, la estima de profesores y superiores, la aceptación y estima de compañeros, y la conducta prosocial. A nivel del autoconcepto social, los hombres de primeros semestres del programa de pregrado en Filosofía obtuvieron menores promedios que los estudiantes de Licenciatura en educación física, Historia y Licenciatura en Ciencias Sociales. Se encontró que los estudiantes de últimos semestres del programa de pregrado en Filosofía, obtuvieron menores promedios que los estudiantes de los programas de Licenciatura en educación física y medicina. No se encontraron diferencias a nivel del desempeño en este factor para las mujeres de primeros y últimos semestres al comparar los diferentes pregrados.

Los resultados encontrados permiten suponer la influencia de la formación orientada en la carrera, en el desarrollo de un mayor autoconcepto social, en especial en carreras consideradas de la línea de profesiones inscritas en las Ciencias Sociales y de la salud, como lo serian programas de pregrado como: Historia, Medicina, Licenciatura en Ciencias Sociales y Licenciatura en educación física en cuanto a lo largo de la carrera los estudiantes de dichos programas evidencian mejores promedios en este factor.

Este hallazgo coincide con los resultados encontrados en una investigación realizada con 691 estudiantes de la ciudad de Temuco que tuvo como objetivo determinar los niveles de autoconcepto, autoeficacia académica y bienestar psicológico en una muestra de estudiantes universitarios chilenos, en la que se encontró que los estudiantes de programas de Licenciatura en educación presentaban mejor autoconcepto social que el resto de estudiantes, estudio desde el cual se consideró que este mejor desempeño a nivel del factor social parecía relacionarse con el perfil profesional declarado para cada carrera universitaria de la que provenían los estudiantes (Veliz Burgos, \& Apodaca - Urguijo, 2012b).

A nivel del autoconcepto social los estudiantes de Medicina de últimos semestres, presentan mejores promedios que los de Filosofía, probablemente desde el efecto que tiene la formación en la disciplina sobre el desarrollo de motivos de tipo altruista y humanitario para la permanencia y finalización de la carrera en el marco de un programa de pregrado con un alta vocación social. Dichos resultados coinciden con los hallazgos de un estudio realizado con 129 estudiantes de medicina de Zaragoza, que tuvo como objetivo analizar las distintas características que definen la decisión de estudiar Medicina y el tipo de motivos por los cuales los estudiantes universitarios permanecen y finalizan la carrera, en el que se encontró que en el caso de los estudiantes de medicina se evidencian fuertes motivos para ingresar y permanecer en la carrera por razones altruistas y humanitarias, entre las que prima la dedicación a los demás y el compromiso con las personas, siendo la vocación social más fuerte a medida que se avanza en la carrera (Soria, Guerra, 
Giménez \& Escanero, 2006; Nunes-Baptista, Rigotto, Ferrari-Cardoso \& Marín-Rueda, 2012; Troncoso-Pantoja, Garay-Lara \& Sanhueza-Muñoz, 2016).

Para el caso de los hombres de primeros y últimos semestres del programa de Filosofía se obtienen menores promedios en este factor, que el resto de programas evaluados, desempeño que probablemente se asocia a la especie de crisis existencial que parece ser propia de la decisión de estudiar Filosofía y de las mismas prácticas pedagógicas y didácticas en las que se considera como orientación esencial el desarrollo de la actitud de filosofar. En tal sentido, se ha señalado que el filosofar es entendido como el uso libre de la razón, alejado de la imitación y repetición de lo que otros han razonado. El filosofar tiene que ver con preguntar, pero no toda pregunta es filosófica, para que una pregunta sea filosófica, deben tener una correlación, un vínculo vital con quien las plantea y con el problema, no interesa tanto el desarrollo de aptitudes sociales, como si el uso libre de la razón sobre aquello que disponga y apasione a cada uno (Manaced-Rey, 2012). Así entendido, en relación con el autoconcepto social, pueden evidenciarse menores promedios, probablemente relacionados con hallazgos investigativos que señalan que en la enseñanza y el aprendizaje del "filosofar" se supone la necesidad de lograr un cambio subjetivo en la forma de conceptualizar la propia realidad en la interacción social, que implica un pensar dialógico compartido, en el que confluyen las miradas problematizadoras particulares (Colella, 2014).

No se identificaron diferencias para el caso de las mujeres de primeros y últimos semestres en torno al autoconcepto social, lo que parece indicar que la formación profesional disciplinar impartida en las carreras, parece estar impactando poco el factor social del autoconcepto, hallazgo que se distancia de los resultados encontrados por Amezcua \& Pichardo (2000) en un estudio realizado con 1235 adolescentes que tuvo como objetivo profundizar en el análisis de las diferencias de género en relación con el autoconcepto, quienes reportan un mejor autoconcepto social para el caso de las mujeres.

El autoconcepto emocional, hace referencia a la percepción que tiene el sujeto de su estado emocional y de sus respuestas a situaciones específicas. El factor hace referencia a la percepción general del propio estado emocional (soy nervioso, me asusto con facilidad, etc) y a la percepción del propio control emocional en situaciones más específicas donde la otra persona implicada es de un rango superior (cuando me preguntan, me hablan, etc). Los estudiantes de primeros semestres del programa de Filosofía, obtienen mayores promedios que los estudiantes de la Licenciatura en Educación física, Licenciatura en Ciencias Sociales, Historia y Medicina a nivel del autoconcepto emocional. No se evidencian diferencias significativas a nivel de este factor, entre los estudiantes hombres de últimos semestres, ni entre las estudiantes mujeres de primeros y últimos semestres.

Dichos resultados se distancian de lo encontrado en un estudio realizado con 120 estudiantes de una Universidad de España que tuvo como objetivo comprobar si la inteligencia emocional percibida actúa como factor moderador del ajuste emocional, estudio en el que se encontraron diferencias en la percepción, desde el cual se indicó que las mujeres presentaron una mayor tendencia a pensar y atender a sus emociones que los hombres, aunque no se encontraron diferencias en la comprensión y la regulación (Jiménez \& López, 2008). Así mismo, los resultados encontrados, se distancian de los hallazgos presentados en otro estudio realizado por Amezcua \& Pichardo (2000) 
en que se evalúo el autoconcepto de 1235 adolescentes con el objetivo de profundizar en el análisis de las diferencias de género existentes en sus niveles de autoconcepto, en el que se encontró que los jóvenes presentaron mayores niveles de autoconcepto global y emocional, mientras que sus compañeras destacaron en el autoconcepto familiar.

Resulta de interés que los estudiantes hombres de primeros semestres del programa de Filosofía, presenten mejores promedios en el autoconcepto emocional que el resto de programas, dicho resultado parece validar lo planteado por Bravo-Montenegro (2012) al considerar que se requiere cierta actitud inquisitiva frente a la pregunta por la vida misma y por las propias emociones para estudiar filosofía. Los resultados presentados coinciden con lo señalado en un estudio que tuvo como objetivo analizar las diferencias de género y edad en las distintas dimensiones del autoconcepto en una muestra de 1414 estudiantes chilenos, en el que se encontró que los hombres, puntuaron más alto en la dimensión de Estabilidad Emocional (Vicent, Lagos-San Martín, Gonzálvez, Inglés, García-Fernández, \& Nieves-Gomis, 2015).

El autoconcepto familiar se entiende como la percepción que tiene el sujeto de su implicación, participación e integración en el medio familiar. El significado de este factor se relaciona con el afecto y la confianza establecida en la relación con los padres, así como con la percepción de felicidad y apoyo que se percibe en la familia y el hogar. En la presente investigación no se encontraron diferencias significativas a nivel de este factor entre estudiantes de primeros y últimos semestres, ni diferencias de género en los diferentes programas evaluados, hallazgo que contribuye a confirmar lo planteado por García \& Musitu (2009) al señalar que al llegar a la Universidad, el autoconcepto de los varones permanece en unos promedios equivalentes a la línea estable de las mujeres.

Así mismo, puede señalarse que los resultados encontrados se distancian de los hallazgos presentados por Véliz-Burgos \& Apodaca-Urquijo (2012a) quienes encontraron en una investigación realizada con 691 estudiantes universitarios diferencias en el autoconcepto al revisar la formación profesional, desde sus hallazgos establecen que en cuanto al área disciplinar, los estudiantes de carreras relacionadas con el área psicosocial presentan mayores promedios en los factores emocional y familiar. Así mismo, puede indicarse que los resultados encontrados frente al autoconcepto familiar, se distancian de los hallazgos encontrados en una investigación realizada con 1.112 personas que tuvo como objetivo determinar la relación entre el autoconcepto y el contexto familiar, en el que se encontró que las mujeres presentan un pobre autoconcepto familiar, por encima de los hombres, resultado en el estudio asociado a que la muestra evaluada reportó haber ser víctima de violencia psicológica (Gutiérrez-Quintanilla, 2014).

Puede señalarse que el autoconcepto físico se entiende como el factor que hace referencia a la percepción que tiene el sujeto de su aspecto físico y condición física. En la presente investigación los hombres de primeros y últimos semestres del programa de Licenciatura en educación física, obtienen mayores promedios que los estudiantes de los programas de Historia, Licenciatura en Ciencias Sociales, Medicina y Filosofía y letras. En el caso de las mujeres de primeros y últimos semestres, no se evidenciaron diferencias significativas entre los pregrados evaluados. 
Los resultados encontrados parecen confirmar el efecto de la disciplina y la formación profesional sobre el autoconcepto, en especial en relación con el factor físico, en la medida en que potenciar dicho factor parece estar directamente relacionado con el propósito de formación de dicha carrera profesional. Se considera que La Licenciatura en Educación física de la Universidad de Caldas tiene como principal interés, formar licenciados idóneos, que aporten al mejoramiento de la calidad de vida y a la construcción de tejido social, mediante el fomento de la actividad física, la práctica deportiva y el adecuado uso del tiempo libre, lo que se hace evidente en los mayores promedios obtenidos por los estudiantes de dicha carrera respecto a las demás carreras profesionales incluidas en la presente investigación (Universidad de Caldas, 2013). Dichos hallazgos confirman también, los resultados encontrados por Amezcua \& Pichardo (2000)en sus estudios al reportar que en relación con la diferencias de género en estudiantes universitarios, los hombres presentan mayor nivel de autoconcepto físico que las mujeres, y se distancia de lo señalado en otros estudios, en los que se reconoce la importancia del autoconcepto físico para las mujeres adolescentes (Fernández, González, Contreras \& Cuevas, 2015).

Por último puede señalarse que para el caso de las mujeres, se evidencia que a medida que se avanza en la carrera profesional se incrementa el promedio a nivel de autoconcepto académicolaboral, lo que coincide con los resultados encontrados en algunos de sus estudios por García \& Musitu (2009), García \& Musitu (2014), en los que reportan que el autoconcepto académico-laboral es superior en las mujeres que en los valores y que la cima de este factor se alcanza con la finalización de la carrera profesional y con la integración en el mundo laboral.

En el caso de los hombres, a medida que se va finalizando la carrera profesional, se incrementa el promedio obtenido a nivel del autoconcepto académico-laboral en el caso de los estudiantes de medicina y el autoconcepto físico a nivel de los estudiantes del programa de Licenciatura en Educación física. Lo que parece coincidir con los hallazgos de Véliz-Burgos \& Apodaca-Urquijo (2012a) al señalar que en sus investigaciones se observa que los estudiantes universitarios hombres presentan mayor nivel de autoconcepto físico y que los estudiantes de salud presentan mejor autoconcepto académico.

En general pareció ser una constante que en los estudiantes tanto hombres, como mujeres, quedaran ubicados por encima del promedio de los baremos propuestos por García \& Musitu (2009), lo que pone de relieve la necesidad de realizar estudios de estandarización de la prueba para población universitaria colombiana a fin de tener unos parámetros normativos del instrumento más pertinentes de acuerdo con el contexto nacional y regional.

Nota de Autores

El presente artículo se deriva de la investigación denominada: "Estandarización de pruebas cognitivas y caracterización del desempeño en jóvenes universitarios de la ciudad de Manizales", realizada en convenio entre investigadores de la Universidad de Caldas y la Universidad de Manizales. 


\section{REFERENCIAS}

Acosta, M. (1998). Creatividad, motivación y rendimiento académico. Granada: Aljibre.

Amezcua, J. \& Pichardo, M. (2000). Diferencias de género en autoconcepto en sujetos adolescentes. Anales de Psicología, 16(2), 207-214. Recuperado de: http://www.um.es/analesps/v16/v16_2/10-16_2.pdf

Avendaño, B., Jiménez, M. \& Senior, D. (2008). Caracterización de un grupo de estudiantes que obtuvo altos puntajes en el examen de calidad de la Educación superior, ECAES, 2003-2006. Suma Psicológica, 15(2), 355-384. DOI: http://dx.doi.org/10.14349/sumapsi2008.44177

Bravo-Montenegro, E. (2012). ¿Aprender filosofía o aprender a filosofar? Reflexiones en torno a la enseñanza de la filosofía. Memorias tercer congreso colombiano de filosofía. Bogotá: Editorial Sociedad Colombiana de Filosofía.

Burns, R. (1990). El autoconcepto. Teoría, medición, desarrollo y comportamiento. Bilbao: Ediciones Egea. Recuperado de: https://books.google.com.co/books/about/El_autoconcepto.html?id=Mbq6PQAACAAJ\&redir_esc= y

Bustos, V., Oliver, A. \& Galiana, L. (2015). Validación del autoconcepto Forma 5 en universitarios peruanos: una herramienta para la Psicología Positiva. Psychology/Psicologia Reflexão e Critica, 28(4), 690697. DOI: http://dx.doi.org/10.1590/1678-7153.201528406

Byrne, B. (1984). The general/academic self-concept nomological network: A review of construct validation research. Review of Educational Research, 54(3), 427-456. DOI: https://doi.org/10.3102/00346543054003427

Byrne, B. (1986). Self-concept/academic achievement relations: An investigation of dimensionality, stability and causality. Canadian Journal of Behavioural Science, 18(2), 173-186. DOI: http://dx.doi.org/10.1037/h0079982

Byrne, B.M. \& Shavelson, R.J. (1986). On the structure of adolescent self-concept. Journal Educational Psychology, 78(6), 474-481. DOI: http://dx.doi.org/10.1037/0022-0663.78.6.474

Cataño, D., Restrepo, S., Portilla, N. \& Ramírez, H. (2008). Autoestima y sexualidad en adolescentes: validación de una escala. Revista de investigaciones Andina, 10(16), 57-66. Recuperado de: http://www.scielo.org.co/pdf/inan/v10n16/v10n16a05.pdf

Cazalla-Luna, N. \& Morelo, D. (2013). Revisión teórica sobre el autoconcepto y su importancia en la adolescencia. Revista Electrónica de Investigación y Docencia (REID), 10, 43-64. Recuperado de: https://revistaselectronicas.ujaen.es/index.php/reid/article/viewFile/991/818

Clark, A., Clemes, H. \& Bean, R. (2000). Cómo desarrollar la autoestima en adolescentes. Madrid: Editorial Debate. 
Colella, L. (2014). El problema filosófico y el problema de la enseñanza de la Filosofía. Aportes desde la noción de identidad y universalismo de Alain Badiou. Revista Cuestiones de Filosofía, 16, 213-226. DOI: https://doi.org/10.19053/01235095.2401

Cordero-Tapia, O. \& Durán, J. (2015). El autoconcepto en estudiantes de educación general básica con bajo rendimiento académico. (Tesis de pregrado en Psicología). Universidad de Cuenta, Ecuador. Recuperado de: http://dspace.ucuenca.edu.ec/bitstream/123456789/22319/1/Tesis.pdf

Costa, S. \& Tabernero, M. (2012). Rendimiento académico y autoconcepto en estudiantes de educación secundaria obligatoria según el género. Revista Iberoamericana de Psicología y Salud, 3(2), 175-193. Recuperado de: https://www.researchgate.net/publication/277261268

Díaz, A. (2009). Diseño estadístico de experimentos. 2da. edición. Medellín: Editorial Universidad de Antioquia. Recuperado de: https://books.google.com.co/books?isbn=9587142640

Elexpuru, I., Villardón, L. \& Yániz, C. (2013). Identificación y desarrollo de valores en estudiantes universitarios. Revista de Educación, 362, 1-18. Doi: https://doi.org/10.4438/1988-592X-RE-2011362-158

Esnaola, I., Goñi, A. \& Madariaga, J. (2008). El autoconcepto: Perspectivas de investigación. Revista de Psicodidáctica, 13(1), 179-194. Recuperado de: http://www.ehu.eus/ojs/index.php/psicodidactica/article/view/231/227

Esnaola, I., Rodríguez, A. \& Goñi, E. (2011). Propiedades psicométricas del cuestionario de Autoconcepto AF5. Anales de Psicología, 27(1), 109-117. Recuperado de: http://www.redalyc.org/html/167/16717018013/

Esteve, J. (2005). Estilos parentales, clima familiar y autoestima física en adolescentes. España: Editorial Universidad de Valencia. Recuperado de: http://hdl.handle.net/10803/10184

Fernández-Zabala, A., Goñi, E., Rodríguez-Fernández, A. \& Goñi, A. (2015). Un nuevo cuestionario en castellano con escalas de las dimensiones del autoconcepto. Revista Mexicana de Psicología, 32(2), 149-159. Recuperado de: http://www.redalyc.org/resumen.oa?id=243045364005

Fernández, J., González, I., Contreras, O. \& Cuevas, J. (2015). Relación entre imagen corporal y autoconcepto físico en mujeres adolescentes. Revista Latinoamericana de Psicología, 47(1), 25-33. DOI: https://doi.org/10.1016/S0120-0534(15)30003-0

Fleming, J.S. \& Courteney, B.E. (1984). The dimensionality of self-esteem: II. Hierarchical facet model for revised measurement scales. Journal of Personality and Social Psychology, 46(2), 404-421. DOI: http://dx.doi.org/10.1037/0022-3514.46.2.404

Fleming, J.S. \& Watts, W.A. (1980). The dimensionality of self-esteem: Some results for collage sample. Journal of Personality and Social Psychology, 39(5), 921- 929. DOI: http://dx.doi.org/10.1037/00223514.39.5.921

Garaigordobil, M., Aliri, J. \& Fontaneda, I. (2009b). Subjective psychological well-being: Gender differences, relations with personality dimensions and predictor variables. Behavioral Psychology Psicología 
Conductual, $\quad$ 17(3), 543-559. Recuperado de: https://www.researchgate.net/publication/215731847

García-Cruz, A. (2016). Familia, educación y la construcción de la identidad y del autoconcepto en niños escolares. Revista Psicoeducativa: reflexiones y propuestas, 2(3), 40-46. Recuperado de: http://psicoeducativa.iztacala.unam.mx/revista/index.php/rpsicoedu/article/view/22/74

García-Fernández, J., Inglés, C., Díaz-Herrero, A., Lagos-Sanmartín, N., Torregrosa, M. \& Gonzálvez, C. (2016). Capacidad predictiva de la autoeficacia académica sobre las dimensiones del autoconcepto en una muestra de adolescentes chilenos. Revista Estudios sobre Educación, 30, 31-50. DOI: https://doi.org/10.15581/004.30.31-50

García, L. (2004). Actores cognitivos, motivacionales y autoconcepto en relación al rendimiento académico. Lima: UNMSM.

Garcia, F. \& Gracia, E. (2010). What is the optimum parental socialisation style in Spain? A study with children and adolescents aged 10-14 years. Infancia y Aprendizaje, 33(3), 365-384. Doi: https://doi.org/10.1174/021037010792215118

García, F. \& Musitu, G. (1999). Autoconcepto Forma 5, AF5. Publicaciones de Psicología Aplicada. Madrid: TEA Ediciones, S.A. Recuperado de: http://www.web.teaediciones.com/Ejemplos/AF5_Manual_2014_extracto.pdf

García, F. \& Musitu, G. (2009). AF5 Autoconcepto Forma 5. Madrid: Ediciones TEA. Recuperado de: http://www.web.teaediciones.com/Ejemplos/AF-5_Manual_2014_extracto.pdf

García, F. \& Musitu, G. (2014). AF5 Autoconcepto Forma 5. Madrid: Ediciones TEA. Recuperado de: http://www.web.teaediciones.com/Ejemplos/AF-5_Manual_2014_extracto.pdf

García, P.G., Sarmiento, A.M. \& Martínez, R.G. (2011). Differences in self-concept by gender in the adolescence: Construction and validation of an instrument. Liberabit, 17(2), 139-146. Recuperado de: http://revistaliberabit.com/es/revistas/RLE_17_2_diferencias-en-el-autoconcepto-por-sexoen-la-adolescencia-construccion-y-validacion-de-un-instrumento.pdf

González-Pienda, J.A., Núñez-Pérez, J.C. \& Valle-Arias, A. (1992). Procesos de comparación externa/interna, autoconcepto, y rendimiento académico. Revista de Psicología general y aplicada, 45(1), 73-81. Recuperado de: https://dialnet.unirioja.es/servlet/articulo?codigo=2377592

González, M. \& Tourón, J. (1992). Autoconcepto y rendimiento escolar: sus implicaciones en la motivación y en la autorregulación del aprendizaje. Pamplona: EUNSA. Recuperado de: https://dadun.unav.edu/handle/10171/21388

Goñi-Palacios, E. (2009). El autoconcepto personal: Estructura interna, medida y variabilidad. Vitoria: Servicio Editorial de la Universidad del País Vasco. Recuperado de: https://addi.ehu.es/bitstream/handle/10810/12241/go\%C3\%B1i\%20palacios.pdf?sequence=1

Guitrón, A. (2013). Aproximación fenomenológica al autoconcepto personal y social del adulto joven mexicano. Revista electrónica en ciencias sociales y humanidades apoyadas por nuevas tecnologías, 
2(1), 69-88. Recuperado de: http://chat.iztacala.unam.mx/cshat/index.php/cshat/article/view/44/38

Gutiérrez-Quintanilla, J. (2014). El contexto familiar asociado al autoconcepto y al desempeño académico de los jóvenes de educación media de San Salvador. Revista Entorno, 55, 41-50. Recuperado de: http://biblioteca.utec.edu.sv/entorno/index.php/entorno/article/view/17/18

Hoeve, Y., Jansen, G. \& Roodbol, P. (2014). The nursing profession: public image, self-concept and professional identity. Journal of Advanced Nursing, 70(2), 295-309. DOI: https://doi.org/10.1111/jan.12177

Ibarra, E., Armenta, M. \& Jacobo, H. (2014). Autoconcepto, estrategias de afrontamiento y desempeño profesional docente. Revista de currículum y formación del profesorado, 18(1), 223-239. Recuperado: http://www.ugr.es/ recfpro/rev181COL2.pdf

Ji, J. (2015). Understanding science museumeducators in China and their self-concept as professionals. (Tesis de Doctorado). University of British Columbia, Estados Unidos. Recuperado de: http://hdl.handle.net/2429/54877

Jiménez, M. \& López, E. (2008). El autoconcepto emocional como factor de riesgo emocional en estudiantes universitarios. Diferencias de género y edad. Boletín de Psicología, 93, 21-39. Recuperado de: https://www.uv.es/seoane/boletin/previos/N93-2.pdf

Machargo, J. (1989). El profesor y el autoconcepto de sus alumnos. Madrid: Escuela Española.

Malo, S., Bataller, S., Casas, F., Gras, M.E. \& González, M. (2011). Análisis psicométrico de la escala multidimensional de autoconcepto AF5 en una muestra de adolescentes y adultos de Cataluña. Psicothema, 23(4), 871-878. Recuperado de: http://www.psicothema.com/pdf/3969.pdf

Manaced-Rey, E. (2012). Filosofía y filosofar en Educación. El reto de la filosofía: filosofar. III Congreso Colombiano de Filosofía. Bogotá: Editorial Sociedad Colombiana de Filosofía. DOI: http://dx.doi.org/10.26620/uniminuto.polisemia.7.11.2011.10-19

Marsh, H. (2005). Self-concept theory, measurement and research into practice: the role of self-concept in educational psychology. The education section of the British psychological society. Recuperado de: https://www.researchgate.net/publication/242268806_SelfConcept_Theory_Measurement_and_ Research_into_Practice_the_role_of_self-concept_in_educational_psychology

J.M. Suls (Ed) (1993), The self in social perspective (59-98p).

Hillsdale, NJ, US: Lawrence Erlbaum Associates. Marsh, H. \& Craven, R. (2006). Reciprocal effects of selfconcept and performance from a multidimensional perspective. Beyond seductive pleasure and unidimensional perspectives. Perspectives on Psychological Science, 1(2), 133-163. https://doi.org/10.1111/j.1745-6916.2006.00010.x

Marsh, H.W. \& Ayotte, V. (2003). Do multiple dimensions of self-concept become more differentiated with age? The differential distinctiveness hypothesis. Journal of Educational Psychology, 95(4), 687-706. DOI: http://dx.doi.org/10.1037/0022-0663.95.4.687 


\section{Diana Marcela Montoya Londoño, Victoria Eugenia Pinilla Sepúlveda, Carmen Dussán Luberth}

Marsh, H.W., Craven, R.G. \& Debus, R. (1991). Self-concepts of young children aged 5 to 8: Their measurement and multidimensional structure. Journal of Educational Psychology 83(3), 377-392. DOI: http://dx.doi.org/10.1037/0022-0663.83.3.377

Marsh, H.W., Byrne, B.M. \& Shavelson, R. J. (1988). A Multifaceted academic self-concept: Its hierarchical structure and its relation to academic achievement. Journal of Educational Psychology, 80(3), 366380. DOI: http://dx.doi.org/10.1037/0022-0663.80.3.366

Mestre, M.V. \& Pérez-Delgado, E. (1994). Desarrollo del autoconcepto en el ámbito familiar. En E. PérezDelgado, Familia y educación. Relaciones familiares y desarrollo personal de los hijos. Valencia: Generalist Valencia.

Moritz, K., Read, M., Clark, R., Callahan, C. \& Albaugh, S. (2009). Grade and Gender Differences in Gifted Students Self-Concepts. Journal for the Education of the Gifted, 32(3), 340-367. Doi: https://doi.org/10.4219/jeg-2009-862

Musitu, G., García, F. \& Gutiérrez, M. (1997). AFA (Autoconcepto Forma-A). Madrid: TEA Ediciones. Recuperado de: https://www.uv.es/garpe/C_L_/022_Musitu_et_1991.pdf

Musitu, G., Román, J.M. \& Gracia, E. (1988). Familia y educación. Prácticas educativas de los padres y socialización de los hijos. Barcelona: Labor.

Nunes-Baptista, M., Rigotto, D., Ferrari-Cardoso, H. \& Marín-Rueda, F. (2012). Soporte social, familiar y autoconcepto: Relación entre los constructos. Revista Psicología desde el Caribe, 29(1), 1-18. Recuperado de: http://rcientificas.uninorte.edu.co/index.php/psicologia/article/viewFile/1916/9192

Ospina, D. (2001). Introducción al muestreo. Santa Fe de Bogotá: Universidad Nacional de Colombia.

Parra, C., Restrepo, G., Usuga, O., Castañeda, E., Estrada, P., M., Uñates, E., Gil, A. \& Mendoza, R. (2015). Relación del Autoconcepto y del acompañamiento psicopedagógico con el rendimiento académico en estudiantes de primer semestre de ingeniería. Revista Ingeniería \& Sociedad, 9, 40-66. Recuperado de: http://aprendeenlinea.udea.edu.co/revistas/index.php/ingeso/article/view/25046/20380

Peralta-Sánchez, F. \& Sánchez-Roda, M. (2003). Relaciones entre el autoconcepto y el rendimiento académico en alumnos de educación primaria. Revista electrónica de investigación psicoeducativa y psicopedagógica, 1(1), 95-120. Recuperado de: http://www.investigacionpsicopedagogica.org/revista/new/ContadorArticulo.php?7

Polo-Sánchez, M., \& López-Justicia, M. (2012). Autoconcepto de estudiantes universitarios con discapacidad visual, auditiva y motora. Revista Latinoamericana de Psicología, 44(2), 87-98. DOI: http://dx.doi.org/10.14349/rlp.v44i2.1035

Ramírez, S. (2012). Impacto del Autoconcepto académico en los participantes en las comunidades virtuales. (Tesis de doctorado en Filosofía). Universidad Autónoma Nuevo León, Monterrey-México. Recuperado de: http://eprints.uanl.mx/3164/1/1080237539.pdf 
Ramos-Díaz, E., Rodríguez-Fernández, A., Fernández-Zabala, A., Revuelta, A. \& Zuazagoitia, A. (2016). Apoyo social percibido, autoconcepto e implicación escolar de estudiantes adolescentes. Revista de Psicodidáctica, 21(2), 339-356. DOI: https://doi.org/10.1387/RevPsicodidact.14848

Revuelta-Revuelta, L., Rodríguez-Fernández, A., Ruiz de Azúa-Ormaza, U. \& Ramos-Díaz, E. (2015). Autoconcepto multidimensional: medida y relaciones con el rendimiento académico. Revista Internacional de Evaluación y Medición de la Calidad Educativa, 2(1), 12-25. Recuperado de: http://journals.epistemopolis.org/index.php/calidadeducativa/article/view/987/551

Salvador-Ferrer, C. (2012). Influence of Emotional Intelligence in Self-Concept. International Journal of Learning \& Development, 2(1), 232-240. DOI: https://doi.org/10.5296/ijld.v2i1.1256

Saura-Calixto, P. (1995). La educación del autoconcepto: Cuestiones y propuestas. Estrategias, técnicas y actividades para el autoconocimiento, entrenamiento en habilidades sociales, desarrollo de expectativas, estilo atribucional y autocontrol. Murcia: Publicaciones Universidad de Murcia.

Shavelson, R. J., Hubner, J. \& Stanton, J. (1976). Self-concept: Validation of construct interpretations. Review of Educational Research, 46(3), 407-411. DOI: https://doi.org/10.3102/00346543046003407

Sheskin, D. (2007). Handbook of parametric and nonparametric statistical procedures. Boca Ratón, Florida: Chapman \& Hall/CRC, Taylor \& Francis Group. Recuperado de: http://library.mpibberlin.mpg.de/toc/z2007_770.pdf

Silva-Ramos, L. (2016). Cuestionario de autoconcepto físico en universitarios de Trujillo metropolitano. Revista de Psicología, 18(1), 9-25. Recuperado de: http://repositorio.ucv.edu.pe/handle/UCV/189

Soria, M., Guerra, M., Giménez, I. \& Escanero, J. (2006). La decisión de estudiar medicina: Características. Revista de Educación médica, 9(2), 91-97. Recuperado de: http://scielo.isciii.es/pdf/edu/v9n2/original4.pdf

Strein, W. (1993). Advances in research on academic self-concept: Implications for school psychology. School Psychology Review, 22(2), 273-284. Recuperado de: http://www.nasponline.org/publications/periodicals/spr/volume-22/volume-22-issue-2/advancesin-research-on-academic-self-concept-implications-for-school-psychology

Tajfel, H. \& Turner, J.C. (1986). The social identity theory of intergroup behavior. In S. Worchel \& W. Austin (Eds.), Psychology of intergroup relations. Chicago: Nelson-Hall. Recuperado de: http://web.mit.edu/curhan/www/docs/Articles/15341_Readings/Intergroup_Conflict/Tajfel_\%26_ Turner_Psych_of_Intergroup_Relations_CH1_Social_Identity_Theory.pdf

Torres-Monge, L. (2016). Inteligencia emocional, autoconcepto y autoestima a través de la educación plástica. (Tesis de pregrado). Universidad de Valladolid, España. Recuperado de: https://uvadoc.uva.es/bitstream/10324/21404/1/TFG-L1437.pdf

Troncoso-Pantoja, C., Garay-Lara, B. \& Sanhueza-Muñoz, P. (2016). Percepción de las motivaciones en el ingreso a una carrera del área de la salud. Revista Horizonte médico, 16(1), 55-61. Recuperado de: http://www.horizontemedicina.usmp.edu.pe/index.php/horizontemed/article/view/396/315 
Universidad de Caldas (2013). Oferta académica Facultad de Ciencias para la Salud. Programa de Licenciatura en Educación con énfasis en Educación Física, Recreación y Deportes. Página web Universidad de Caldas. Disponible en: http://aspirantes.ucaldas.edu.co/licenciatura-en-educacionfisica-recreacion-ydeporte/

Urquijo, S. (2002). Auto-concepto y desempeño académico en adolescentes. Relaciones con sexo, edad e institución. Psico-USF, 7(2), 211-218. Recuperado de: http://www.scielo.br/pdf/pusf/v7n2/v7n2a10.pdf

Valenzuela-Zambrano, B. \& López-Justicia, M. (2015). Autoconcepto de estudiantes universitarios chilenos con discapacidad. Diferencias en función del género. Revista nacional e internacional de educación inclusiva, $\quad$ 8(3), 153-170. Recuperado de: http://www.revistaeducacioninclusiva.es/index.php/REl/article/view/96/93

Vélez, A., Doner, A. \& Sandoval, S. (2016). Relación entre autoconcepto, autoeficacia académica y rendimiento académico en estudiantes de salud en Puerto Montt, Chile. Revista Educadi, 1(1), 97109. http://dx.doi.org/10.7770/educadiv1N1-art1003

Véliz, B.A. (2010). Dimensiones del autoconcepto en estudiantes chilenos: Un estudio psicométrico. Bilbao: Universidad del País Vasco. Recuperado de: https://www.educacion.gob.es/teseo/imprimirFicheroTesis.do?idFichero=19823

Véliz-Burgos, A. \& Apodaca-Urquijo, P. (2012a). Dimensiones del autoconcepto de estudiantes chilenos: Un estudio psicometrico. Revista Educativa Hekademos, 11(5), 47-58. Recuperado de: https://dialnet.unirioja.es/servlet/articulo?codigo=4059766

Véliz-Burgos, A. \& Apodaca-Urquijo, P. (2012b). Niveles de autoconcepto, autoeficacia académica, y bienestar psicológico en estudiantes universitarios de la ciudad de Temuco. Revista Salud \& Sociedad, 3(2), 131-150. http://dx.doi.org/10.22199/S07187475.2012.0002.00002

Vicent, M., Lagos-San Martín, N., Gonzálvez, C., Inglés, C., García-Fernández, J. \& Nieves-Gomis, N. (2015). Diferencias de género y edad en autoconcepto en estudiantes adolescentes chilenos. Revista de Psicologia, 24(1), 1-16. DOI: http://dx.doi.org/10.5354/0719-0581.2015.36752

Vicuña, L. (2004). La motivación de logro y el autoconcepto en estudiantes de la Universidad Nacional Mayor de San Marcos. Revista de Investigación en Psicología del Instituto de Investigaciones Psicológicas, 7(2), 136-149. DOI: http://dx.doi.org/10.15381/rinvp.v7i2.5127

Walpole, R., Myers, R.H., Myers, S.L. \& Ye, K. (2012). Probabilidad y estadística para ingeniería y ciencias. México: Pearson Educación. Recuperado de: https://vereniciafunez94hotmail.files.wordpress.com/2014/08/8va-probabilidad-y-estadisticapara-ingenier-walpole_8.pdf 